\title{
Perspektiven alter(n)sgerechter Betriebs- und Tarifpolitik
}

Resümiert man die hierzulande seit etlichen Jahren geführte Diskussion zum Umgang mit den erwarteten Folgen erwerbsdemografischen Wandels für Betriebe und Beschäftigte, dann stellt man eine beträchtliche Lücke zwischen verbaler Problematisierung und Debattenstand einerseits und der Wirklichkeit praktischen betrieblichen Handelns andererseits fest. Der Beitrag leuchtet diese Lücke für drei Kernbranchen der deutschen Wirtschaft genauer aus: die Metall- und Elektroindustrie, die Chemie- und Pharmaindustrie und den Einzelhandel. Darüber hinaus diskutiert er Möglichkeiten und Grenzen alter(n)sgerechter Betriebs- und Tarifpolitik. ${ }^{\bullet}$

KNUT TULLIUS, JOHANNES FREIDANK, JOHANNES GRABBE, JÜRGEN KÄDTLER, WOLFGANG SCHROEDER

\section{Einleitung}

Im Zusammenhang mit Befunden und Prognosen zur Bevölkerungsentwicklung in Deutschland wird in jüngerer Zeit verstärkt über gesellschaftliche Handlungsoptionen zur Bewältigung der damit verbundenen Herausforderungen diskutiert. Letztere werden vor allem in Finanzierungsproblemen bei der Alterssicherung und in einem tatsächlichen oder vermeintlichen Fachkräftemangel ausgemacht. Mit Blick auf das Erwerbsarbeitssystem rücken in diesem Zusammenhang zunehmend die Regulierung des Altersübergangs sowie die Arbeits- und Beschäftigungsbedingungen ins Zentrum der Aufmerksamkeit. Wenn Erwerbstätige länger arbeiten sollen und müssen - so lautet seit Langem der Tenor in Arbeits- und Sozialwissenschaft -, sind Arbeitsbedingungen in den Betrieben so zu gestalten, dass dies den Beschäftigten auch faktisch möglich ist. An wissenschaftlich-praktischen Gestaltungs- und Handlungsempfehlungen zu solcherart alters- und alternsgerechten Arbeitsbedingungen besteht kein Mangel. Allerdings zeigt die bisherige empirische Forschung, dass daraus in der betrieblichen Praxis bislang wenig folgt. Ziel dieses Beitrags ist es zum einen, diese Lücke zwischen Debattenund betrieblicher Umsetzungsrealität auf der Grundlage eines gerade abgeschlossenen Forschungsprojekts ${ }^{2}$ genauer zu vermessen. Dazu werden die Problemlagen von Betrieben und die Handlungsorientierungen und Perspekti- ven betrieblicher und überbetrieblicher Akteure in drei Kernbranchen der deutschen Wirtschaft dargestellt: der Metall- und Elektroindustrie (M\&E), der Chemie- und Pharmaindustrie und dem Einzelhandel. Zum anderen werden mögliche Erklärungen für die skizzierte Kluft zwischen Handlungsbedarf und seiner Umsetzung sowie Ansätze tarifvertraglicher Regulierung zu ihrer Schließung diskutiert.

Nach einem kurzen Resümee des Forschungsstandes (Abschnitt 2) zeigen wir nachfolgend zunächst die Problemlagen auf, die betriebliche Akteure in den drei von uns untersuchten Branchen im Zusammenhang mit der erwerbsdemografischen Entwicklung sehen (3). Im Anschluss (4) betrachten wir die Verbreitung alters- und alternsgerechter betrieblicher Gestaltungsmaßnahmen in den

Wir danken den beiden anonymen Gutachtern der WSI-Mitteilungen für ihre kritischen Hinweise zu einer Vorfassung dieses Beitrages.

2 Die vom Lehrstuhl „Politisches System der BRD - Staatlichkeit im Wandel“ der Universität Kassel in Kooperation mit dem SOFI Göttingen durchgeführte Untersuchung „Potenziale altersdifferenzierter und alternsgerechter Betriebsund Tarifpolitik" wurde von der „Initiative Neue Qualität der Arbeit" (INQA) gefördert und von der Bundesanstalt für Arbeitsschutz und Arbeitsmedizin (BAuA) begleitet. Der Forschungsbericht zum Projekt ist in der Reihe "INQA-Berichte" erschienen (Freidank et al. 2011). 
Betrieben, wobei sich branchendifferenziert unterschiedlich ausgeprägte Defizite zeigen. In Abschnitt 5 diskutieren wir die Gründe für diese Defizite. Abschnitt 6 greift die tarifpolitische Diskussion anhand beispielhafter Tarifregelungen in der Chemie- und Pharmaindustrie auf.

\section{Erwerbsdemografischer Wandel - Erkenntnisstand und Umsetzungs- lücken}

Seit mehr als 20 Jahren sind bestimmte Trends der Bevölkerungsentwicklung in der Mehrzahl europäischer Gesellschaften, mit diesen Trends verbundene Problemlagen sowie mögliche Lösungsansätze unter der Überschrift „Demografischer Wandel“ Gegenstand wissenschaftlicher und politischer Debatten. ${ }^{\mathbf{3}}$ Das Erwerbspersonenpotenzial ist bereits seit 1995 rückläufig (Statistisches Bundesamt/ WZB 2011, S. 14). Es wird nach vorliegenden Prognosen ab etwa 2020 verstärkt schrumpfen und durchschnittlich deutlich altern (Statistisches Bundesamt 2009; Fuchs/Zika 2010). Als Resultat wird unter anderem von einem Zuwachs des Anteils der nicht mehr erwerbstätigen Alten an der Bevölkerung und von einem deutlich steigenden Durchschnittsalter auch der Belegschaften in den Betrieben ausgegangen (Bellmann et al. 2003, 2007). Sozial-, renten- und arbeitsmarktpolitische Neuregelungen der letzten Jahre, die mit den Finanzierungsproblemen der zunehmenden „Alterslast" begründet werden, verstärken den betrieblichen Alterungseffekt dadurch, dass die bisherigen Möglichkeiten eines flexibleren Altersübergangs - ggf. auch eines vorzeitigen Erwerbsausstieges - deutlich begrenzt oder versperrt wurden. ${ }^{\circledR}$ Als Folgen dieser erwerbsdemografischen Entwicklung werden mit Blick auf hiesige Unternehmen unter anderem Nachwuchs- und Fachkräftemangel, ${ }^{\boldsymbol{}}{ }^{\text {ein }}$ Anstieg der Krankheitskosten und ein Nachlassen, wenn nicht gar ein Verlust der Wettbewerbs- und Innovationsfähigkeit prognostiziert (Brasche/Wieland 2000; Kaufmann 2005). Stand der Alternsforschung ist allerdings, dass nicht das Alter(n) per se zu einer Verringerung der Arbeits- und Leistungsfähigkeit der Beschäftigten führt bzw. führen muss (Behrend 2002; BMFSFJ 2005). Vielmehr sind im Erwerbslebenslauf akkumulierte berufs- und tätigkeitsspezifische (Fehl-)Belastungen und unzureichende Möglichkeiten des Kompetenzerwerbs und -erhalts, aber auch das individuelle Gesundheitsverhalten, maßgeblich verantwortlich für Einschränkungen der Arbeits- und Beschäftigungsfähigkeit Älterer. Zumindest unter den gegebenen Arbeitsbedingungen und physischen wie psychosozialen Belastungen scheint es für Erwerbstätige in vielen Berufen und Tätigkeitsfeldern nahezu ausgeschlossen, die (künftige) Regelaltersgrenze von 67 Jahren zu erreichen (vgl. u. a. Bäcker et al. 2009; Heien et al. 2008; Trischler/Kistler 2010).
Unter Arbeits- und Sozialwissenschaftlern besteht Konsens darüber, dass die Schaffung alters- und alternsgerechter Arbeitsbedingungen ein - wenn nicht der - Schlüssel zur Bewältigung der angesprochenen betrieblichen und individuellen Probleme ist (vgl. u. a. Ilmarinen/Tempel 2002; Morschhäuser 2006; Lorenz/Schneider 2008). Altersgerecht sind Arbeitsbedingungen dann, wenn sie die besonderen Anforderungen und Bedürfnisse bereits älterer Beschäftigter (gemeint ist i.d.R. die Altersgruppe der über 50- oder über 55-Jährigen) etwa bei der Gestaltung der Arbeits- und Lernumgebung, der Arbeitszeit oder auch der Leistungsanforderungen berücksichtigen; alternsgerecht sind sie dann, wenn sie ,über die ganze Erwerbsbiografie so gestaltet sind, dass keine Spätfolgen auftreten und die Beschäftigten gesund, motiviert und produktiv das Rentenalter erreichen und auch danach noch ihren Ruhestand gesund erleben" (Kistler 2008, S. 40).

Mittlerweile liegt eine Vielzahl von Handlungsempfehlungen und Praxisbeispielen dazu vor, wie alterns- und altersgerechte Arbeits- und Beschäftigungsbedingungen konkret gestaltet sein müssten (vgl. unter vielen: HBS 2009; INQA 2005; Kocka/Staudinger 2009; Matthäi/Morschhäuser 2009). Gemeinsam ist diesen Empfehlungen die Betonung einer Verknüpfung oder Integration unterschiedlicher Konzepte und Maßnahmen - gelegentlich auch als „AgeManagement“ bezeichnet -, die sich insgesamt sechs Gestaltungsfeldern zuordnen lassen:

(1) Personalpolitik: Rekrutierungspraxis; Personalplanung und -entwicklung; Analyse von Alters- und Qualifikationsstrukturen

(2) Arbeitsorganisation und -gestaltung: Lernförderlichkeit von Arbeit; altersdifferenzierte Leistungsanforderungen

(3) Arbeitszeitgestaltung und Work-Life-Balance

(4) Qualifizierung, Weiterbildung und Lebenslanges Lernen

(5) Gesundheitsmanagement

(6) Führung und Unternehmenskultur.

3 Vgl. etwa die Ende der 1980er Jahre initiierten, seit 1993 regelmäßig erscheinenden „Altenberichte" der Bundesregierung; der mittlerweile sechste Bericht ist Ende 2010 erschienen. Der fünfte Altenbericht setzte den Schwerpunkt auf „Potenziale des Alters in Wirtschaft und Gesellschaft” (vgl. BMFSFJ 2005).

4 Die hauptsächlichen Stichworte sind bekannt: die sukzessive Heraufsetzung der gesetzlichen Regelaltersgrenze für den abschlagsfreien Rentenbezug auf 67 Jahre bis Ende 2029; die Abschaffung der „58er-Regelung“ im Sozialgesetzbuch Drittes Buch (SGB III) zum 1.1.2008, die älteren Arbeitslosen einen leichteren Arbeitslosengeldbezug ermöglichte und die Beendigung der staatlichen Förderung der Altersteilzeit nach dem AltTZG von 1996 zum 1.1.2010. Frehmel (2011, S. 9) spricht mit Blick auf das Ergebnis dieser Neuregelungen von einer "gesetzlichen Entflexibilisierung" des Altersübergangs.

5 Zu Kontroversen um diesen Punkt vgl. bereits Bellmann et al. 2003 sowie jüngst Baethge et al. 2010; Brenke 2010. 
Während öffentliche und wissenschaftliche Debatte, Konzeptentwicklung und Umsetzungsempfehlungen ein beachtliches Niveau aufweisen, legt die empirische Forschung den Schluss nahe, dass insgesamt nur wenige Betriebe etwas für den Erhalt und die Entwicklung der Arbeits- und Beschäftigungsfähigkeit ihrer älteren Beschäftigten und ihrer strukturell alternden Belegschaften tun (Backes-Gellner 2009; Bellmann et al. 2007; Brussig et al. 2009; Commerzbank 2009; Kistler 2008; Moraal et al. 2009; Morschhäuser 2006). Nur in einer Minderheit der Betriebe finden sich arbeits- und personalpolitische Maßnahmen, die sich gezielt an Ältere (50+) richten - mit Altersteilzeit als der am weitesten verbreiteten „Maßnahme“ -, während etwa arbeitsgestalterische Elemente („altersgemischte Arbeitsgruppen“) oder ergonomische Maßnahmen („Ausstattung der Arbeitsplätze") so gut wie nicht vorkommen. Bei der Rekrutierung werden Jüngere deutlich vorgezogen, während Ältere häufig nur unter bestimmten Voraussetzungen, etwa bei Erhalt von Lohnkostenzuschüssen, eingestellt werden (vgl. Bellmann et al. 2003, S. 144f.). Die Forschung zu Qualifizierung und Weiterbildung belegt, dass Betriebe in den letzten Jahren im Allgemeinen zunehmend weniger in Weiterbildung und Kompetenzentwicklung investiert haben und dass Ältere sofern nicht bereits gut qualifiziert - sowie andere „Risikogruppen“ (Geringqualifizierte, Migranten, Teilzeit arbeitende Frauen) signifikant seltener an betrieblicher Weiterbildung teilnehmen (Autorengruppe 2010; BMBF 2011; Leber/Möller 2008; Öztürk 2009). Ähnliche Selektivitätseffekte gelten auch für die sogenannte Lernförderlichkeit der Arbeit, d.h. die im unmittelbaren Arbeitsprozess gegebenen Möglichkeiten, die eigenen Kompetenzen und Fähigkeiten weiterzuentwickeln (vgl. Baethge/Baethge-Kinsky 2004).

\section{Branchenspezifische Problemlagen und -wahrnehmungen: Empirische Befunde}

Vor dem Hintergrund dieses Forschungs- und Debattenstands untersuchten wir in den Jahren 2009 und 2010 die branchenspezifische Relevanz des „Demografieproblems“ in der Chemie- und Pharmaindustrie, in der Metall- und Elektroindustrie (M\&E-Industrie) und im Einzelhandel. Ziel unseres Projekts war es, Genaueres über (Einflussfaktoren auf) die Verbreitung (oder Nicht-Verbreitung) von Gestaltungsmaßnahmen einer alter(n)sgerechten Arbeits- und Personalpolitik in den Betrieben dieser drei Branchen zu erfahren. Angesichts der vor Beginn des Projekts intensiv geführten tarifpolitischen Debatte und gerade abgeschlossener neuer Tarifverträge in der Eisen- und Stahlindustrie und der Chemieindustrie - aber auch angesichts der Rezession von 2007ff. - sollten auch die Perspektiven für vergleichbare „Demografietarifverträge“ in der M\&E-Industrie und im Ein- zelhandel sowie gegebenenfalls deren Verallgemeinerungsfähigkeit ausgelotet werden. Empirische Grundlage der hier präsentierten Befunde sind vor allem Ergebnisse einer eigenen standardisierten Befragung von Geschäfts- und Personalleitungen $(\mathrm{N}=474)$ und Betriebsräten $(\mathrm{N}=311){ }^{\bullet}$ Darüber hinaus führten wir elf betriebliche Kurzfallstudien durch, bei denen wir insgesamt 42 Expertinnen und Experten, vor allem aus Personalleitung und Betriebsrat, interviewten. Auf Branchen- und Verbandsebene (Gewerkschaften, Arbeitgeberverbände) führten wir insgesamt zwölf Experteninterviews.

Zunächst ein Blick auf prägnante Unterschiede und Gemeinsamkeiten der untersuchten Branchen.

\subsection{Altersstrukturen}

Bereits hinsichtlich der Altersstrukturen weisen die Metallund Elektro-, die Chemie- und Pharmaindustrie sowie der Einzelhandel deutliche Unterschiede und damit „objektiv“ unterschiedliche Lagen auf: Die aktuellen Altersstrukturprofile in den beiden Industriebranchen sind durch eine „Rechtsgipfeligkeit“ und überdurchschnittlich viele Beschäftigte in den Altersgruppen 40+ gekennzeichnet - auch im Vergleich zur Gesamtbeschäftigung in Deutschland (Abbildung 1). Demgegenüber zeigt die Altersstruktur im Einzelhandel eine „zweigipfelige“ Form mit überdurchschnittlich vielen jüngeren und deutlich weniger älteren Beschäftigten. Dieses Charakteristikum des Einzelhandels liegt in der (nach wie vor) hohen Ausbildungsquote, in dem hohen Frauenanteil und in den „weiblichen“ Erwerbsverlaufsmustern - woraus sich u. E. die „Delle“ in der Kohorte der 30- bis 40-Jährigen erklärt - begründet. Die Abbildung zeigt auch, dass die rentennahe Altersgruppe der 60- bis 64-Jährigen mit 2,9 \% im Einzelhandel, 3,6 \% in der Chemie- und Pharmaindustrie und 3,9\% in der M\&E-Industrie aktuell nur einen sehr geringen Anteil an den Beschäftigten ausmacht.

6 Es handelt sich bei den im Text präsentierten Befragungsergebnissen um gewichtete Daten für die drei Branchen in Betrieben mit mehr als fünf Beschäftigten. Die Gewichtung dient dazu, die Aussagekraft der Befragungsergebnisse so zu verbessern, dass wir repräsentative Aussagen treffen können. Aufgrund geringer Fallzahlen in der Chemie-/Pharmabranche ( $N=29$ Personaler; $N=15$ Betriebsräte) sind die dortigen Ergebnisse jedoch sehr vorsichtig zu interpretieren, auch im Vergleich zu den beiden anderen Branchen. Die Gewichtung im Managersample berücksichtigt Branchenmerkmale und $\mathrm{Be}$ triebsgröße, bei der Gewichtung des Betriebsrätesamples wurde ergänzend auf Daten des Wirtschafts- und Sozialwissenschaftlichen Instituts (WSI) der Hans-Böckler-Stiftung zurückgegriffen, die uns freundlicherweise zur Verfügung gestellt wurden. Insgesamt wurden 4.500 Betriebe (dort jeweils Geschäfts- und Personalleitungen einerseits, Betriebsräte andererseits) auf der Grundlage einer repräsentativen Adressziehung der Bundesagentur für Arbeit angeschrieben und um Teilnahme an einer Online-Befragung gebeten. Um die Ausschöpfung zu erhöhen, wurden CATI-gestützteTelefoninterviews "nachgeschoben“. Durch dieses Verfahren sind gewisse Verzerrungen, z. B. durch Selektivitätseffekte, nicht auszuschließen (vgl. Freidank et al. 2011). 
ABB.

\section{Altersstrukturen sozialversicherungspflichtig Beschäftigter}

Anteile der Beschäftigten einzelner Altersgruppen an allen Beschäftigten in Prozent

- Gesamt*

- Chemie/Pharma

Metall \& Elektroindustrie

Einzelhandel

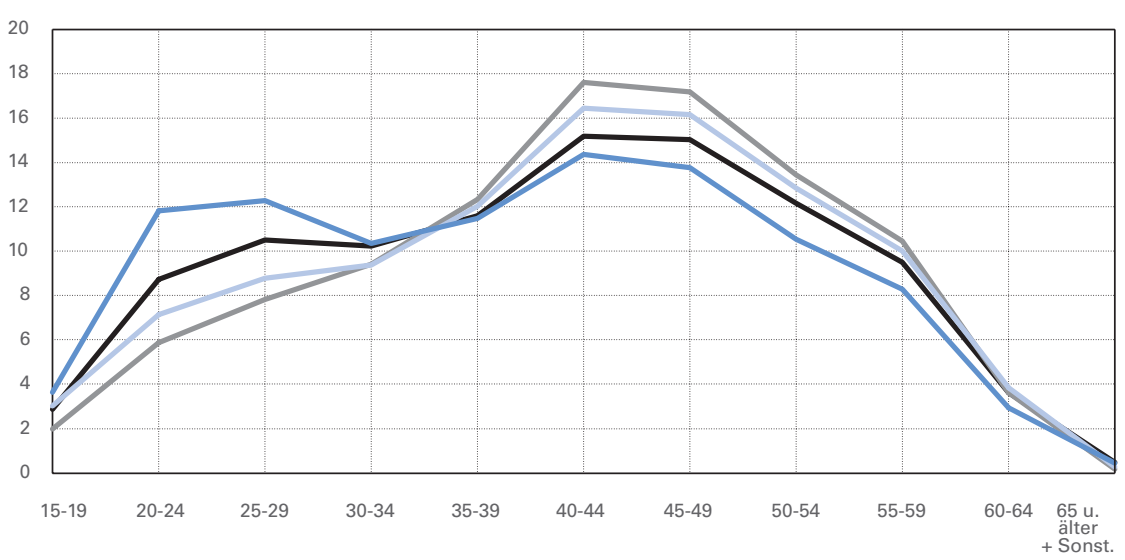

*Gesamt = alle sozialversicherungspflichtig Beschäftigten in Deutschland.

Quelle: Bundesagentur für Arbeit 2010, Stichtag 30.06.2009; Berechnungen und Darstellung der Autoren. rend Einzelhandelskaufleute im Bereich der psychischen Arbeitsanforderungen („emotionale Anforderungen“) einen signifikant niedrigeren ( $=$ schlechteren) Wert aufweisen als die Beschäftigten in den beiden Industriebranchen. Daten der Deutschen Rentenversicherung zeigen, dass - nimmt man die Anteile von Erwerbsminderungsrenten zum groben Maßstab - das Niveau des vorzeitigen Verlustes der Arbeitsfähigkeit in den Hauptberufen unserer Branchen nach wie vor beträchtlich ist: So geht fast jeder fünfte Chemiebetriebswerker (Tätigkeitsschlüssel 141) oder Maschinenschlosser (273), mehr als jeder vierte Kunststoffverarbeiter (151), knapp jeder vierte Schweißer (241) und mehr als jeder fünfte KFZ-Instandsetzer (281), aber auch Verkäufer (682), wegen verminderter Erwerbsfähigkeit vorzeitig in Rente (DRV Bund 2011). Diese Zahlen markieren jedoch nur die Spitze des Eisbergs gesundheitsbedingten vorzeitigen Ausstiegs aus dem Erwerbsleben, d.h. sie unterzeichnen das tatsächliche Niveau der Fehlbelastungswirkungen. Dies liegt zum einen daran, dass die Statistik der Rentenversicherung nur den zuletzt ausgeübten Beruf vor Rentenbeginn erfasst. ${ }^{8}$ Generelle Aussagen zu Berufen, Tätigkeitsprofilen bzw. zu Branchen sind bei einer solchen Querschnittsbetrachtung daher nur eingeschränkt möglich. Zum anderen wird nur etwas mehr als jeder zweite Antrag auf Erwerbsminderungsrente anerkannt, sodass der Verdacht naheliegt, dass deutlich mehr Ältere aus gesundheitlichen Gründen aus dem

$(0$ Die Indexwerte für körperliche Anforderungen lauten jeweils: Metallerzeuger/-bearbeiter: 37; Maschinen- und Fahrzeugbauer: 46; Maschinisten und zugehörige Berufe: 41. Die Berufsgruppen der Chemiearbeiter/ Kunststoffverarbeiter (Indexwert 50) und der Einzelhandelskaufleute (Indexwert 56) liegen im untersten Bereich der Kategorie „mittelmäßige Arbeit” (vgl. DGB-Index Gute Arbeit GmbH 2009).

8 Beispiel: War jemand 30 Jahre als Schweißer und -z.B. aus gesundheitlichen Gründen - nur die letzten Monate vor Rentenbeginn im Lager beschäftigt, so erscheint diese Person als Lagerarbeiter in der Statistik und nicht als Schweißer.

TABELLE 1

\section{Arbeitsinduzierte körperliche Beschwerden von Erwerbstätigen}

Angaben in Prozent

\begin{tabular}{lccc}
\hline & $\begin{array}{c}\text { Metall- und } \\
\text { Elektroindustrie }\end{array}$ & $\begin{array}{c}\text { Chemie- und } \\
\text { Pharmaindustrie }\end{array}$ & Einzelhandel \\
\hline $\begin{array}{l}\text { Während/unmittelbar nach der Arbeit Schmerzen im } \\
\text { unteren Rücken, Kreuzschmerzen }\end{array}$ & 39,9 & 40,5 & 49,0 \\
\hline $\begin{array}{l}\text { Während/unmittelbar nach der Arbeit Schmerzen im } \\
\text { Nacken-, Schulterbereich }\end{array}$ & 40,4 & 39,9 \\
\hline $\begin{array}{l}\text { Schmerzen in den Beinen, Füßen, } \\
\text { geschwollene Beine }\end{array}$ & 18,9 & 20,2 & 48,7 \\
\hline
\end{tabular}


Erwerbsleben ausscheiden, dann jedoch etwa in Arbeitslosigkeit entlassen werden (vgl. Brussig 2010, S. 13).

Die Betriebe der M\&E-Industrie und der Chemie- und Pharmaindustrie sind also mit demografisch stark alternden Belegschaften konfrontiert; die Beschäftigten unterliegen einem nach wie vor hohen Niveau körperlicher und psychischer Belastungen. Teilweise erhebliche Zweifel an deren Vermögen, ihre Arbeitsfähigkeit bis zum regulären Renteneintrittsalter zu erhalten, sind daher angebracht. Wachsende Probleme für Betriebe wie für Erwerbstätige sind auch deshalb absehbar, weil Arbeit in allen drei Branchen, wenn auch in jeweils unterschiedlicher Weise, einer erheblichen, teils in den Arbeitsfolgen widersprüchlichen Veränderungsdynamik unterworfen ist, auf die die arbeitssoziologische Forschung der letzten Jahre vielfach hingewiesen hat. Die einschlägigen Stichworte lauten: (Informations-)Technisierung, sich wandelnde und steigende Wissens- und Kompetenzanforderungen, permanenter organisatorischer Wandel und „Projektifizierung“, Re-Kontraktualisierung von Arbeit, „Subjektivierung“, Intensivierung und Verdichtung, Flexibilisierung, (Re-)Standardisierung. Der in den letzten Jahren beobachtete Anstieg insbesondere von psychosozialen Erkrankungen wird unter anderem auf diese Entwicklungsdynamik zurückgeführt (vgl. etwa BAuA 2010, S. 5; DAK 2011).

\subsection{Problemdruck und Ursachenbewertung}

Betrachtet man die Bewertung des demografischen Wandels aus Sicht der Betriebe, dann zeigt sich zunächst, dass - in der Wahrnehmung des Managements - nur eine Minderheit aktuell vom demografischen Wandel betroffen ist ( $\mathrm{Ab}$ bildung 2). Für die Zukunft wird - wiederum branchenübergreifend - eine spürbar stärkere Betroffenheit ausgemacht.

Am deutlichsten werden Auswirkungen des demografischen Wandels in der Chemie- und Pharmaindustrie konstatiert - sowohl gegenwärtig als auch für die Zukunft. Als am wenigsten spürbar wird dieser in Betrieben des Einzelhandels eingeschätzt. Fragt man in den Branchen danach, in welcher Art und Weise sich der demografische Wandel konkret bemerkbar macht, dann kann man etwas generalisierend sagen, dass in der Chemie- und Pharmaindustrie neben Fachkräftemangel bzw. Know-how-Verlust die mit Arbeitsbelastungen verbundenen Probleme im Vordergrund stehen (Tabelle 2). Auch in der M\&E-Industrie wird eher ein Wissens- und Know-how-Verlust beobachtet. Im Einzelhandel spielen beide Aspekte eine deutlich geringere Rolle. Diese Branchendifferenzen reflektieren Besonderheiten der Arbeitsbedingungen und Tätigkeitsanforderungen sowie der Beschäftigungsformen und personalwirtschaftlichen Praktiken und deren Dynamik in den vergangenen 20 Jahren. In der M\&E-Industrie und zunehmend auch in der Chemie- und Pharmaindustrie bedeutet dies: qualifizierte Produktions- und Angestelltentätigkeiten unter regelmäßig körperlich und/oder psychisch belastenden Arbeitszeit- und Prozessbedingungen in Vollzeitarbeitsverhältnissen, in denen sich Arbeitsbelastungen im
ABB. 2

\section{Betroffenheit vom demografischen Wandel}

Angaben „trifft eher zu“ und „trifft völlig zu“ in Prozent

- Zukunft Gegenwart

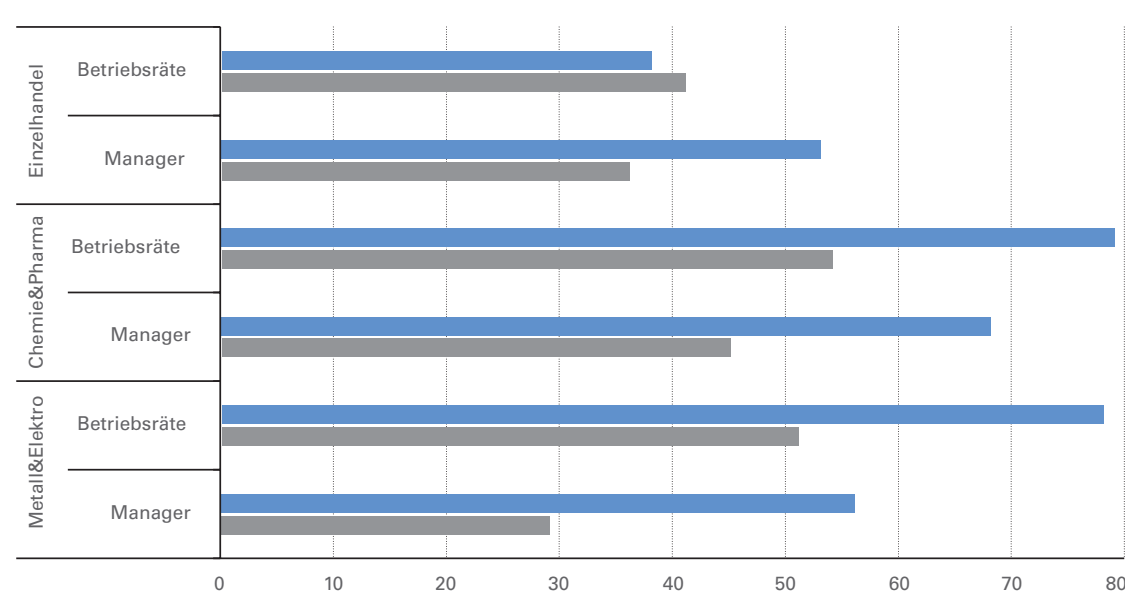

Quelle: Befragung und Darstellung der Autoren.

Erwerbsverlauf akkumulieren. Im Einzelhandel heißt das: ein verbreiteter Trend zu geringer qualifizierter Dienstleistungsarbeit, die zwar mit erheblichen körperlichen und psychosozialen Belastungen verbunden sein kann, die aber nicht zuletzt wegen der typischen, überwiegend „weiblichen" Erwerbsverlaufsmuster (Teilzeitarbeit, Familienpausen) und anhaltend hoher Ausbildungszahlen nicht zu gravierenden Problemen für die Personalbewirtschaftung der Betriebe führen. ${ }^{\bullet}$

Markanter als die Branchendifferenzen sind die unterschiedlichen Einschätzungen von Betriebsrat und Management: Betriebsräte bekunden zum einen eine deutlich höhere betriebliche Betroffenheit als das Management (Ausnahme Einzelhandel). Zum anderen unterscheiden sich die Einschätzungen auch in Bezug auf die mit dem demografischen Wandel verbundenen Auswirkungen in den Betrieben deutlich (Tabelle 2): Während Personaler hauptsächlich Wissensoder Know-how-Verluste problematisieren, spielen diese seitens der Betriebsräte zwar auch eine Rolle, deutlich stärker werden aber - mit Ausnahme des Handels - Folgen von Fehlbelastungen angesprochen. Vergleichbares lässt sich auch mit Blick auf die personalwirtschaftlichen Probleme Älterer (,50+“) zeigen: Während das Management vor allem den Bereich der Weiterbildungsbeteiligung und -neigung sowie - selbstkritisch? - fehlende betriebliche Entwicklungs-

(9 Eine Ausnahme stellen aufgrund derTätigkeiten (Kommissionierung, Verladearbeiten) und Arbeitsbedingungen (Schichtarbeit, [männliche] Vollzeitarbeit) hier die Logistikbetriebe des Handels dar. 
TABELLE 2

\section{Die „Top 5“ betrieblicher Probleme heute}

nach Betriebspartei und Branche

Geschäfts- bzw. Personalleitungen

Betriebsräte

\begin{tabular}{|c|c|c|}
\hline \multicolumn{3}{|c|}{ Metall- und Elektroindustrie } \\
\hline 1 & Nachwuchsmangel (58 \%) & Verlust von Erfahrungswissen (70 \%) \\
\hline 2 & Fachkräftemangel (56 \%) & Zunahme von Leistungs- und Tätigkeitseinschränkungen (49\%) \\
\hline 3 & Verlust von Erfahrungswissen (55 \%) & Nachwuchsmangel (34 \%) \\
\hline 4 & Verstärkte Rekrutierung Älterer (42 \%) & Hoher Krankenstand / hohe Fehlzeiten ( $34 \%$ ) \\
\hline 5 & Zunahme von Leistungs- und Tätigkeitseinschränkungen (30 \%) & Verstärkte Rekrutierung Älterer (27 \%) \\
\hline \multicolumn{3}{|c|}{ Chemie- und Pharmaindustrie } \\
\hline 1 & Verlust von Erfahrungswissen ( $58 \%$ ) & Verlust von Erfahrungswissen (76 \%) \\
\hline 2 & Fachkräftemangel (42\%) & Hoher Krankenstand / hohe Fehlzeiten (63\%) \\
\hline 3 & Nachwuchsmangel (42\%) & Zunahme von Leistungs- und Tätigkeitseinschränkungen (38 \%) \\
\hline 4 & Zunahme von Leistungs- und Tätigkeitseinschränkungen ( $40 \%$ ) & Fachkräftemangel (13\%) \\
\hline 5 & Hoher Krankenstand / hohe Fehlzeiten ( $30 \%$ ) & Nachwuchsmangel (13\%) \\
\hline & \multicolumn{2}{|c|}{ Einzelhandel } \\
\hline 1 & Verlust von Erfahrungswissen (50\%) & Verlust von Erfahrungswissen (61 \%) \\
\hline 2 & Nachwuchsmangel (48\%) & Fachkräftemangel (61 \%) \\
\hline 3 & Fachkräftemangel (47 \%) & Hoher Krankenstand / hohe Fehlzeiten (44 \%) \\
\hline 4 & Zunahme von Leistungs- und Tätigkeitseinschränkungen ( 47 \%) & Nachwuchsmangel (44\%) \\
\hline 5 & Führungskräftemangel (44 \%) & Zunahme von Leistungs- und Tätigkeitseinschränkungen (38 \%) \\
\hline
\end{tabular}

Anmerkung: Nur die Gruppe der Befragten, die ihre Betriebe aktuell vom demografischen Wandel betroffen sehen. Hier sei nochmals darauf hingewiesen, dass die Ergebnisse aufgrund geringer Fallzahlen in der Chemie-/Pharmaindustrie sehr vorsichtig zu interpretieren sind.

Quelle: Befragung der Autoren.

möglichkeiten für diese Gruppe als Problemfelder angibt, stehen für die Betriebsräte die Belastungswirkungen und Probleme hinsichtlich der weiteren beruflichen Entwicklung im Betrieb im Vordergrund. Besonders auffallend ist, dass das Thema „psychische Belastungen" als besonderes Problem Älterer bei den Betriebsräten aller drei Branchen unter den häufigsten Nennungen auftaucht, bei den Personalmanagern dagegen überhaupt nicht.

Nach unseren Eindrücken aus den Fallbetrieben kommen zwei Erklärungsmöglichkeiten für diese Unterschiede in Betracht. So haben offensichtlich die unterschiedlichen Funktionen und institutionalisierten Rollen im Betrieb auch unterschiedliche Problemwahrnehmungen und Prioritätensetzungen zur Folge: Personaler haben vor allem Arbeitskräftebeschaffungs- und Kompetenzprobleme im Blick, Betriebsräte agieren stark aus ihrer betrieblichen Schutzfunktion heraus. Darüber hinaus dürften Beschäftigte sehr viel eher geneigt sein, gegenüber dem Betriebsrat psychische (Fehl-)Belastungen zu äußern als gegenüber einem Personaler (d.h. damit verbundene Probleme „offiziell“ $\mathrm{zu}$ machen). In jedem Fall geben diese Befunde einen Hinweis darauf, dass der demografische Wandel und seine Folgen von den betrieblichen Akteuren immer vor dem Hintergrund ihrer Interessenpositionen und konkreten Handlungskontexte wahrgenommen und bewertet werden.

\section{Verbreitung alters- und alterns- gerechter betrieblicher Gestaltungs- maßnahmen}

Im Hinblick auf die Verbreitung von betrieblichen Maßnahmen zum Erhalt und zur Verbesserung der Arbeitsund Beschäftigungsfähigkeit der Erwerbstätigen im demografischen Wandel ${ }^{\oplus}$ haben wir zwischen altersgerechten und alternsgerechten Maßnahmen unterschieden (vgl. Abschnitt 2).

(10) Unsere Befragung zielte hier nicht auf einen unmittelbaren Zusammenhang zu etwaigen „Demografieproblemen“ der Betriebe, sondern wir suchten nach aktuellen Indizien für eine ggf. veränderte Arbeits- und Personalpolitik, die in diesem Sinne auch alters- und alternsgerecht bzw. "demografiesensibel“ sein kann. Nach unserem Kenntnisstand liegt gegenwärtig keine vergleichbare Befragung vor, die ähnlich differenziert angelegt ist. Der Frageblock wurde durch folgenden Hinweis eingeleitet: „Man kann grundsätzlich unterscheiden zwischen betrieblichen Maßnahmen, die die Arbeitsbedingungen aller Beschäftigten im Betrieb - unabhängig von ihrem Alter - verbessern können und solchen Maßnahmen, die sich speziell an ältere Beschäftigte richten.” 


\subsection{Altersgerechte Maßnahmen}

Sieht man sich die Verbreitung von Maßnahmen des ersten Typs an, ${ }^{(1)}$ dann zeigt sich branchenübergreifend (Abbildung 3), dass nur in einer Minderheit der Betriebe altersgerechte Maßnahmen für die Kohorte der über 50-Jährigen existieren.

Am weitesten verbreitet sind arbeitsorganisatorische Maßnahmen, wie z. B. Arbeitsplatzrotation zur Vermeidung einseitiger Belastungen, aber auch hier ist mit leicht über einem Drittel der Betriebe in der M\&E-Industrie das Maximum erreicht. Einen nach wie vor recht hohen Stellenwert besitzt zumindest in der Chemie- und Pharmaindustrie zum Befragungszeitpunkt Ende 2009/Anfang 2010 der Frühausstieg in Form der Blockvariante der Altersteilzeit, ${ }^{(1)}$ während die unverblockte Variante auch dort kaum verbreitet ist. Bei Chemie-/Pharma spielen auch besondere Arbeitszeitregelungen für Ältere eine gewisse Rolle, z.B. aufgrund von Regelungen zur Schichtarbeit. Auffallend ist die vergleichsweise hohe Bedeutung betrieblicher Abfindungsangebote in der M\&E-Industrie und dass überdurchschnittlich viele Einzelhandelsbetriebe die Leistungsanforderungen an Ältere gezielt reduzieren. Generell ist auch zu konstatieren, dass Maßnahmen zur Gesundheitsförderung oder zum Gesundheitsschutz für Ältere in nicht mehr als in jedem zehnten Betrieb verbreitet sind. Und schließlich erweist sich das Aktivitätsniveau der Betriebe hinsichtlich betrieblicher Qualifizierungsangebote, die gezielt die Weiterbildungsbedarfe Älterer adressieren, als gering - ein Befund, der in einem krassen Missverhältnis zu dem gerade auch von Managementseite konstatierten besonderen Weiterbildungsbedarf und fehlenden Entwicklungsperspektiven der Älteren im Betrieb steht. Hier ist auch ein Seitenblick auf die $\mathrm{CVTS}^{{ }^{\circledR}}{ }^{3}$-Zusatzerhebung des BIBB (Moraal et al. 2009) instruktiv, wonach eine Mehrheit der Personaler in allen drei Branchen „spezielle Weiterbildungsmaßnahmen für ältere Beschäftigte für sinnvoll“ hält.

\subsection{Alternsgerechte Maßnahmen}

Betrachtet man die Verbreitung von Maßnahmen des zweiten Typs (Abbildung 4), dann zeichnen unsere Daten ein weniger trübes, wenn auch geteiltes Bild. Weit verbreitet in allen drei Branchen sind non-formale Weiterbildungsangebote, d.h. arbeitsplatznahe Lernformen, wie etwa die klassische Unterweisung durch Vorgesetzte. Auch die formalisierte Weiterbildung findet sich häufig, wobei freilich auffällt, dass nur jeder zweite M\&E-Betrieb solche Lernmöglichkeiten bietet. Flexible Arbeitszeitregelungen, die die Akkumulation von Belastungen abmildern können, gibt es vor allem im Einzelhandel, dort freilich nicht als Konsequenz besonderer Sensibilität für Fragen der Alternsgerechtigkeit, sondern als Ausdruck ökonomisch motivierter Effizienzorientierung unter den Bedingungen einer „Frauenbranche“. Der damit de facto verbundene Belastungsausgleich ist dabei verbreitet mit signifikanten Einschnitten bei anderen As-
ABB. 3

Verbreitung altersgerechter Maßnahmen (50+) nach Branche ${ }^{1}$ 2009/2010²

Angaben in Prozent der Betriebe

- Einzelhandel $\square$ Chemie \& Pharma $\backsim$ Metall \& Elektro

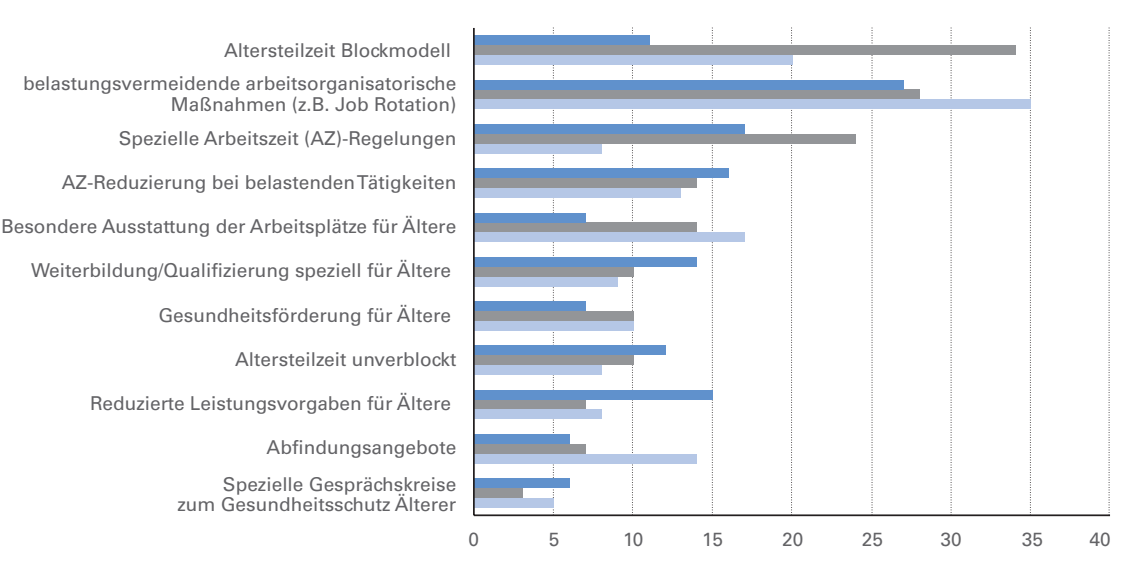

Einzelhandel: $\mathrm{N}=209$; Chemie \& Pharma: $\mathrm{N}=29$; Metall \& Elektro: $\mathrm{N}=223$

2 Die Darstellung beschränkt sich aus Platzgründen auf die Befragung der Manager.

Quelle: Befragung und Darstellung der Autoren.

pekten der Teilhabe in und durch Arbeit verknüpft. Immerhin noch jeder zweite Industriebetrieb gibt an, die Arbeitsplätze seien alternsgerecht gestaltet und vor allem in der M\&E-Industrie finden sich altersgemischte Arbeitsgruppen, während andere arbeitsorganisatorische Maßnahmen seltener sind. Auffallend ist schließlich - daher die Rede vom „geteilten“ Bild -, dass weder Maßnahmen zur Gesundheitsförderung noch solche zur Adressierung der immer mehr an Bedeutung gewinnenden Vereinbarkeitsproblematik (Familie/Pflege und Beruf; Auszeiten bzw. Sabbaticals) in den Betrieben bisher eine nennenswerte Rolle spielen.

\subsection{Zwischenfazit}

Die Bilanz der Verbreitung von altersgerechten arbeits- und personalpolitischen Maßnahmen oder Angeboten in

(1) Die Frage lautete: „Mit Blick auf ältere Beschäftigte (50+): Gibt es im Betrieb eine oder mehrere der folgenden Maßnahmen oder sind diese geplant?" Die Antwortmöglichkeiten lauteten: „weder vorhanden, noch geplant"; „Maßnahme geplant"; "Maßnahmen vorhanden“. Hier sind die Prozentwerte zu "vorhandenen" Maßnahmen angegeben.

(2) Bei diesem „Maßnahmenkatalog“ haben wir aus Vergleichbarkeitsgründen einzelne Dimensionen aus dem IAB-Betriebspanel übernommen (vgl. Bellmann et al. 2003), z.B. die "Altersteilzeit im Blockmodell", die natürlich nicht auf einen längeren Verbleib der Beschäftigten in den Betrieben abzielt, sondern auf das genaue Gegenteil; gleiches gilt für Abfindungsangebote.

(3) Third European Continuing Vocational Training Survey. 
ABB.

\section{Verbreitung alternsgerechter \\ Maßnahmen (50+) nach Branche ${ }^{1}$ 2009/2010² \\ Angaben in Prozent der Betriebe \\ - Einzelhandel $n$ Chemie \& Pharma $\square$ Metall \& Elektro}

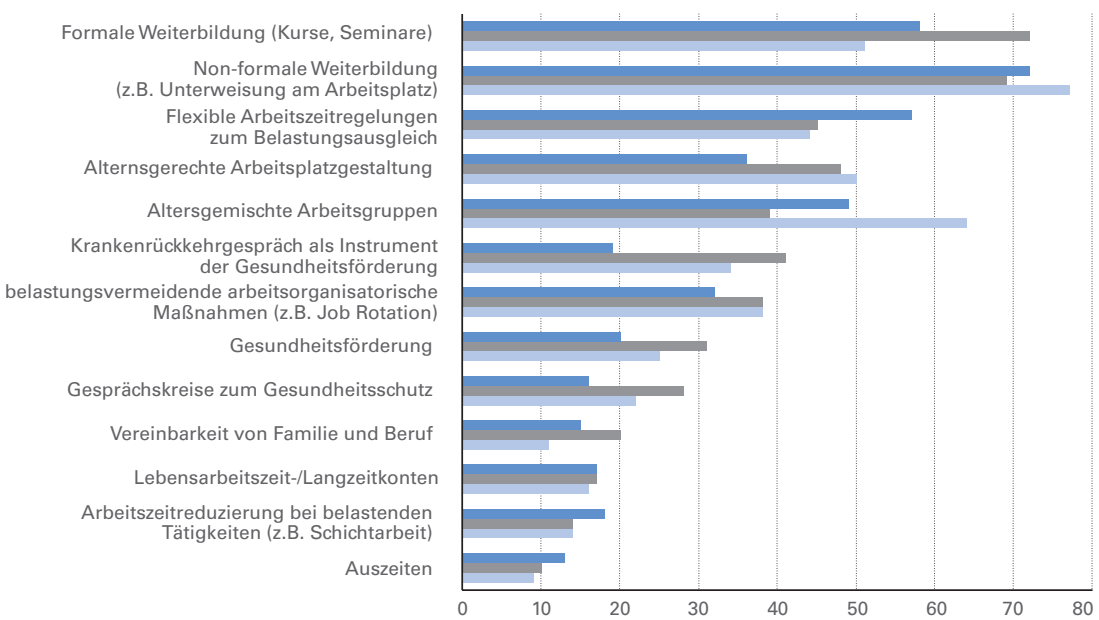

1 Einzelhandel: $\mathrm{N}=208$; Chemie \& Pharma: $\mathrm{N}=29$; Metall \& Elektro: $\mathrm{N}=224$

2 Die Darstellung beschränkt sich aus Platzgründen auf die Befragung der Manager.

Quelle: Befragung und Darstellung der Autoren.

den Betrieben aller drei Branchen ist nach wie vor dürftig. Demgegenüber sind alternsgerechte Maßnahmen teilweise bereits deutlich häufiger umgesetzt. Hier findet sich durchaus ein substanzieller arbeitsgestalterischer Sockel, auf dem eine alternsgerechte Arbeitspolitik aufbauen kann und den es zu verbreitern gilt. Uns interessieren in den beiden abschließenden Abschnitten zum einen die möglichen Gründe für die nach wie vor bestehenden Umsetzungsdefizite, zum anderen die gegenwärtigen Potenziale und Perspektiven der Tarifpolitik für eine Verbesserung der Arbeits- und Beschäftigungsbedingungen im demografischen Wandel.

\section{Zur Lücke zwischen Konzept und Umsetzungsrealität}

Auch unsere Befunde belegen ein Missverhältnis zwischen der Prominenz des Demografiethemas auf der arbeitspolitischen Debatten- und Konzeptebene und seiner Randständigkeit in der betrieblichen Praxis. Das liegt zum Teil daran, dass Ressourcen und Kenntnisse von „Tools“ für ein Altersmanagement - etwa zur Altersstruktur- oder Qualifikationsbedarfsanalyse - fehlen, insbesondere in Klein- und Mittelbetrieben, wie auch andere Studien zeigen (vgl. Latniak et al. 2010; Morschhäuser 2006). Darüber hinaus machen sich Einflüsse der Rezession von 2007ff. bemerkbar: In der Mehrzahl der von ihr betroffenen Betriebe unseres Samples wurden die Prioritäten nicht nur im Management, sondern auch bei den Betriebsräten deutlich zugunsten der Bearbeitung unmittelbar drängender Probleme und zulasten des Themas „Demografie“ verschoben. Darüber hinaus zeigt sich, dass die Feststellung eines Problems auf gesamtgesellschaftlicher Ebene noch nicht dazu führt, dass es für die Handelnden auf der Mikroebene auch zum unmittelbar anzugehenden Problem wird. Das Gefangenendilemma ist der Inbegriff solcher Konstellationen, in denen individuelle Handlungsrationalität das Angehen übergreifender Ziele systematisch blockiert. In eine solche Richtung weist die Humankapitaltheorie (vgl. etwa Becker 1993), nach der Unternehmen grundsätzlich nur dann in z. B. bessere Arbeitsbedingungen oder auch Weiterbildung investieren würden, wenn daraus auch ein (möglichst kurzfristiger) betriebswirtschaftlicher Nutzen zu erwarten sei. Langfristige Perspektiven und Strategien, wie sie alter(n)sgerechte Arbeitsund Betriebspolitik mit dem Ziel einer nachhaltigen Verbesserung der Arbeits- und Beschäftigungsbedingungen voraussetzt, haben es vor dieser Argumentationsfolie eher schwer. Die mögliche Erklärungsrelevanz von Humankapitalansätzen ergibt sich freilich nicht - wie ihre Vertreter meinen - naturgemäß aus individueller Rationalität an sich. Sie ergibt sich vielmehr dadurch und in dem Maße, dass und wie betriebliche Entscheidungsträger sich die entsprechenden Handlungsorientierungen zu eigen machen. Dafür, dass das tatsächlich in verstärktem Maße geschieht, zeichnen Entwicklungen verantwortlich, die als Entwicklung zum Finanzmarktkapitalismus (Windolf 2005) bzw. als Dominantwerden von Finanzmarktrationalität (Kädtler 2010) diskutiert werden. Vertreter der sozialwissenschaftlichen Arbeitsforschung verweisen auf den Zusammenhang zwischen einer dadurch begründeten Kurzfristorientierung und den Grenzen demografieorientierter Personalpolitik (Latniak et al. 2010), der Verdrängung von Ansätzen innovativer Arbeitspolitik durch einen neo-tayloristischen „rollback" (Schumann 1998; vgl. auch Kuhlmann 2009); mancher auch auf eine grundsätzliche Verunmöglichung alterns- und altersgerechter Arbeitsgestaltung und Personalpolitik (Reindl 2009, S. 171). Auch unsere Befunde belegen ein anhaltendes Spannungsverhältnis zwischen einer alters- und alternsgerechten Arbeits- und Beschäftigungspolitik und kapitalistischen Verwertungskalkülen. Wie gesehen, sieht sich eine Mehrheit der Betriebe - aus Arbeitgebersicht - heute gar nicht oder nicht in spürbarer Weise vom demografischen Wandel betroffen. Der aktuelle Handlungsdruck wird offensichtlich als gering eingeschätzt, und solange ihre Wettbewerbs- und Leistungsfähigkeit nicht akut bedroht ist, sehen Betriebe wenig Veranlassung, bessere Arbeits- und Beschäftigungsbedingungen zu schaffen.

Als weiteres gewichtiges Hemmnis für alterns- und v. a. altersgerechte Arbeitspolitik wirkt ein Vorruhestandssyndrom, das im deutsche Beschäftigungssystem seit den 1980er Jahren institutionalisiert worden ist. Als Instrument zur Bewältigung von Massenarbeitslosigkeit und akuten Beschäftigungsproblemen wurde der geregelte, sozialpolitisch flankierte vorzeitige Übergang in den Ruhestand etabliert (Brussig/ 
Knuth 2011), staatlich gefördert und als „Lebensarbeitszeitverkürzung" populär, und zwar gerade in den tariflich und betrieblich gut regulierten Bereichen. Vor dem Hintergrund langfristiger Beschäftigungsabbauprobleme erlangte der Spielraum für „unblutigen“ Personalabbau für Betriebsräte, Gewerkschaften und Beschäftigte eindeutig Priorität, weitreichende humanisierungspolitische Ansätze wie die des Lohnrahmentarifvertrags II für die Metallindustrie in Nordwürttemberg/Nordbaden (Dabrowski et al. 1977) oder das groß angelegte „Projekt Schichtarbeit (PROSA)“ der IG Chemie (vgl. Wolf 1985) traten in den Hintergrund. Den Betrieben eröffneten sich Spielräume für eine aktive Belegschaftsstrukturpolitik bei gleichzeitigem Beschäftigungsabbau sowie für weitere Arbeitsintensivierung, die wiederum einen möglichst frühzeitigen Ausstieg für die betroffenen Beschäftigten zusätzlich wünschenswert machte. Die Institutionalisierung der Lebensarbeitszeitverkürzung gab den Betrieben eine Legitimationsgrundlage dafür, an insgesamt körperlich und psychisch verschleißenden Arbeits- und Beschäftigungsbedingungen nichts Grundlegendes zu ändern. Komplementäre Orientierungen auf Arbeitgeber- und Arbeitnehmerseite am Leitbild Vorruhestand stellen daher einen gewichtigen Faktor dar, der eine Neuorientierung der Arbeits- und Personalpolitiken im betrieblichen Rahmen blockieren kann.

Demografieorientierte Arbeitspolitik, die betriebliche Ansprüche mit denen von Beschäftigten verbinden will, steht damit vor einer doppelten Aufgabe:

- der Revision ihrer auf Altern im Betrieb gerade nicht eingestellten Grundtendenz und

- der Notwendigkeit, für anders orientierte Beschäftigte das Angebot, länger zu arbeiten, wieder attraktiv zu machen.

Darüber hinaus muss sie Optionen für diejenigen bereithalten, die unter den bisherigen Verhältnissen bereits älter geworden sind. Und sie muss weiterhin auf Dauer Frühausstiegsoptionen für solche Beschäftigungsformen, wie z.B. kontinuierliche Schichtarbeit, vorsehen, die auch bei optimaler Gestaltung nicht beeinträchtigungsfrei sind. Effektive Demografieorientierung setzt damit nicht weniger voraus als eine grundlegende Neuausrichtung betrieblicher Arbeits- und Beschäftigungspolitiken, ihre politische und rechtliche Flankierung sowie eine ebenso grundlegende Neufassung arbeitspolitischer Interessenarrangements auf Unternehmens- wie auf gesellschaftlicher Ebene.

\section{Tarifvertragliche Impulse für eine demografieorientierte Arbeits- und Betriebspolitik?}

Alterns- und altersbezogene Regelungen haben in der Tarifpolitik über Vorruhestand und Altersteilzeit hinaus durchaus Tradition (vgl. Bispinck/WSI-Tarifarchiv 2005). Altersbezo- gen sind in erster Linie Kündigungs- und Abgruppierungsschutzregelungen, wobei das Alter allerdings in der Regel erst in Verbindung mit relativ langen Betriebszugehörigkeiten zum Tragen kommt. In einigen Bereichen gibt es nach Lebensalter gestaffelte Urlaubsansprüche oder zusätzliche Freizeitansprüche für ältere Arbeitnehmerinnen und Arbeitnehmer. Senioritäts- und Anciennitätsregelungen bei der tariflichen Bezahlung gibt es seit der Tarifreform im öffentlichen Dienst nicht mehr, sieht man von einem Jugendlichenabschlag für unter 18-Jährige in einigen Branchen einmal ab. Alternsbezogene Regelungen mit dem Fokus auf Belastungsausgleich gibt es in der Form zusätzlicher Urlaubstage für Schichtarbeiterinnen und Schichtarbeiter in der Chemischen Industrie und im Öffentlichen Dienst. Mit Blick auf Qualifikationserhalt bzw. -entwicklung wurden bereits in den 1980er Jahren eine ganze Reihe von Fort- und Weiterbildungsklauseln und seit den 1990er Jahren - zunächst in der Metall-, der Textil- und Bekleidungsindustrie, im Weiteren auch in der Chemischen Industrie - Tarifverträge zur Verankerung von Weiterbildungsansprüchen vereinbart, die zunächst allerdings nur sehr begrenzt praktische Wirkung entfalteten (Bahnmüller et al. 1993, 2006). Durchweg auf freiwillige Vereinbarungen der Betriebsparteien angewiesen, brachen sie sich verbreitet an der skizzierten andersartigen Ausrichtung betrieblicher Personalpolitiken.

Einen tarifpolitischen Neuansatz stellen in diesem Punkt Tarifverträge in der Eisen- und Stahlindustrie aus dem Jahr 2006 (vgl. dazu Katenkamp et al. 2011) und der Chemieindustrie aus dem Jahr 2008 dar, die ausdrücklich auf Demografie Bezug nehmen, und die - das ist das Entscheidende ein Mehr an Verbindlichkeit begründen. Die Umsetzung der Demografietarifverträge in konkrete Maßnahmen bleibt zwar bezogen auf ein breites Spektrum möglicher Regelungsgegenstände an freiwillige Vereinbarungen der Betriebsparteien gebunden. Mit der Verpflichtung, bis zu einem bestimmten Zeitpunkt eine qualifizierte Demografieanalyse der Belegschaften durchzuführen sowie jährlich einen bestimmten Betrag in einen betrieblichen Demografiefonds einzuzahlen, kommen jedoch auch „Einlassungszwang“ und Ressourcen ins Spiel. So fasst der Tarifvertrag (TV) „Lebensarbeitszeit und Demografie" für die Chemieindustrie die Bestimmungen des bestehenden Qualifizierungs-TV mit solchen zu Langzeitkonten, Altersteilzeit, Teilrente, tariflicher Berufsunfähigkeitszusatzversicherung und tariflicher Altersvorsorge zusammen und kombiniert diese mit einem Demografiefonds, in den die Betriebe pro Tarifarbeitnehmer einen jährlichen Betrag von zunächst $300 €$ einzahlen, der sich tarifdynamisch entwickelt. In freiwilligen Betriebsvereinbarungen legen die Betriebsparteien fest, für welche der mögli-

(4) Angesichts bereits eingetretener oder absehbarer gesundheitlicher Beeinträchtigungen war und ist für viele Beschäftigte der frühzeitige Ausstieg die naheliegende, wenn auch häufig unfreiwillig gewählte Option (vgl. Bellmann et al. 2003, S. 141). 
chen Zwecke die Mittel eingesetzt werden sollen. Bei Nichteinigung gehen diese in Betrieben bis 200 Beschäftigten in die tarifliche Altersversorgung, sonst in Langzeitkonten. Mit der bis Ende 2009 durchzuführenden Demografieanalyse erhalten die Betriebsparteien eine umfassende gemeinsame Informationsbasis als Planungs- und Aushandlungsgrundlage.

Unsere Betriebsfallstudien in der Chemieindustrie zeigen, dass dieser letzte Punkt nicht zu unterschätzen ist. Der Zwang zur Demografieanalyse löst im konkreten Fall durchaus handlungsrelevante neue Einsichten und entsprechende praktische Aktivitäten aus. Die praktische Wirkung der zusätzlichen Ressourcenbasis ist gemessen an der Breite vorgesehener Einsatzmöglichkeiten bis auf Weiteres eng fokussiert. Bisher vorliegende Auswertungen der Chemiearbeitgeber (vgl. Schack/Volkwein 2010) und einige Betriebsvereinbarungen zur Verwendung des Demografiebetrags zeigen, dass die oft gewählte Option „Langzeitkonto“ eher nicht auf eine flexible Verteilung der aufgebauten Zeitkonten über die Erwerbslebensphase und insbesondere nicht auf die Qualifizierungsoption zielt, sondern auf eine Block-Verwendung vor dem regulären Renteneintritt. Hier treffen die Betriebsvereinbarungen auf die - übrigens branchenübergreifende - Zustimmung der Betriebsräte unserer Befragung: Die verblockte Altersteilzeit ist für sie die Top-Priorität auf ihrer tarifpolitischen Agenda. Einen zusätzlichen Akzent setzt der „Tarifvertrag über lebensphasengerechte Arbeitszeitgestaltung für die ostdeutsche chemische Industrie“ vom November 2011, der die Einrichtung eines betrieblichen Fonds vorsieht, der aus 2,5 \% der Entgeltsumme des jeweiligen Vorjahrs gespeist wird und alternative Verwendungen für zusätzliche Altersfreizeiten, tarifliche Familienzeiten, Entlastungszeiten für ausgewählte Arbeitnehmergruppen, insbesondere Schichtarbeiter, sowie schließlich Langzeitkonten (auch als Auffangregelung) ermöglicht. In der Option, den Fonds gezielt zur Entlastung besonders belasteter Beschäftigter zu nutzen, liegt ein relevanter Ansatzpunkt für alternsgerechte Arbeitspolitik dort, wo die betreffenden Belastungen nicht auszuschalten sind. Ob das realisiert wird oder schließlich doch überwiegend die Auffangregelung greift, ist derzeit offen. Im Ergebnis ist auch in der Chemieindustrie das Verhältnis zwischen etablierten Vorruhestandsorientierungen und weiterreichenden und notwendigerweise längerfristigen Arbeitsgestaltungsperspektiven in der Schwebe. Demografietarifverträge wie diese bieten Ansatzpunkte für alters- und alternsgerechte Arbeitspolitik in einem wirklich anspruchsvollen Sinne. Die bislang eher enge betriebliche Umsetzung spricht nicht per se gegen die Realisierbarkeit einer solchen Perspektive. Diese setzt aber - wie oben deutlich gemacht eine grundlegende Revision etablierter betrieblicher Personalpolitiken voraus. Tarifpolitik kann ein solches Umsteuern flankieren, durchsetzen kann sie es nicht.

\section{LITERATUR}

Autorengruppe Bildungsberichterstattung (2010): Bildung in Deutschland 2010. Ein indikatorengestützter Bericht mit einer Analyse zu Perspektiven des Bildungswesens im demografischen Wandel, Bielefeld

Backes-Gellner, U. (2009): Beschäftigung älterer Arbeitnehmer im Spiegel bisheriger Forschung, in: Backes-Gellner, U./Veen, St. (Hrsg.): Altern, Arbeit und Betrieb, Stuttgart, S. 11-25

Bäcker, G./Kistler, E./Ebert, A./Trischler, F. (2009): Rente mit 67 - die Voraussetzungen stimmen nicht! Erster Monitoring-Bericht des Netzwerks für eine gerechte Rente, Berlin
Baethge, M./Baethge-Kinsky, V. (2004): Der ungleiche Kampf um das lebenslange Lernen: Eine Repräsentativ-Studie zum Lernbewusstsein und -verhalten der deutschen Bevölkerung, in: edition QUEM, Studien zur beruflichen Weiterbildung im Transformationsprozess, hrsg. von der Arbeitsgemeinschaft Betriebliche Weiterbildungsforschung e.V., Band 18, Münster, S. 9-200

Baethge, M./Laging, J./Philipps, V./Wieck, M. (2010): Perspektiven von Ausbildung und Arbeit im demografischen Wandel, in: Mitteilungen aus dem SOFI 4 (10), S. $1-5$

Bahnmüller, R./Bispinck, R./Schmidt, W. (1993): Betriebliche Weiterbildung und Tarifvertrag. Eine Studie über Probleme qualitativer Tarifpolitik in der Metallindustrie, München

Bahnmüller, R./Fischbach, St./Jentgens, B. (2006): Was nützen und bewirken Qualifizierungstarifverträge?, in: WSI-Mitteilungen 59 (2), S. 71-79, http:// www.boeckler.de/wsi_5880.htm\#results

Becker, G. S. (1993 [1964]): Human Capital. A Theoretical and Empirical Analysis, with Special Reference to Education, Third Edition, Chicago/London Behrend, C. (2002): Erwerbsarbeit Älterer im Wandel - demographische Herausforderungen und Veränderungen der Arbeitsgesellschaft, in: Ders. (Hrsg.): Chancen für die Erwerbsarbeit im Alter. Betriebliche Personalpolitik und ältere Erwerbstätige, Opladen, S. 11-30

Bellmann, L./Hilpert, M./Kistler, E./Wahse, J. (2003): Herausforderungen des demografischen Wandels für den Arbeitsmarkt und die Betriebe, in: Mitteilungen aus der Arbeitsmarkt- und Berufsforschung 36 (2), S. 133-149

Bellmann, L./Kistler, E./Wahse, J. (2007): Demographischer Wandel. Betriebe müssen sich auf alternde Belegschaften einstellen, IAB Kurzbericht (21), Nürnberg Bispinck, R./WSI-Tarifarchiv (2005): Tarifliche Senioritätsregelungen. Eine Analyse von tariflichen Regelungen in ausgewählten Tarifbereichen, hrsg. von der Hans-Böckler-Stiftung, Elemente qualitativerTarifpolitik (59), Düsseldorf

Brasche, U./Wieland, S. (2000): Alter und Innovation: Befunde aus der Beschäftigungsstatistik, in: Vierteljahreshefte zur Wirtschaftsforschung 69 (1), S. 124-141 Brenke, K. (2010): Fachkräftemangel kurzfristig noch nicht in Sicht, in: Wochenbericht des DIW Berlin 77 (46), S. 2-15

Brussig, M. (2010): Künftig mehr Zugänge in Altersrenten absehbar. Gegenwärtig kein Ausweichen in die Erwerbsminderungsrente zu beobachten, Altersübergangsreport 2010-02, Düsseldorf/Berlin/Duisburg/Essen

Brussig, M./Knuth, M. (2011): Alterserwerbstätigkeit und Altersübergang: Alte und neue Herausforderungen, in: Deutsches Zentrum für Altersfragen (Hrsg.): Informationsdienst Altersfragen 38 (4), Juli/August, S. 3-7

Brussig, M./Knuth, M./Wojtkowski, S. (2009): Altersteilzeit: Zunehmende Beschäftigungsbrücke zum späteren Renteneintritt, Altersübergangsreport 2009-02, Düsseldorf/Berlin/Duisburg/Essen

Bundesagentur für Arbeit (BA) (2010): Arbeitsmarkt in Zahlen - Beschäftigungsstatistik. Sozialversicherungspflichtig Beschäftigte nach ausgewählten Wirtschaftszweigen (WZ 2008) und Alter, Statistik Datenzentrum, Nürnberg Bundesanstalt für Arbeitsschutz und Arbeitsmedizin (BAuA) (Hrsg.) (2010): Integration der psychischen Belastungen in die Gefährdungsbeurteilung. Handlungshilfe, Dortmund

Bundesinstitut für Berufsbildung (BIBB) (2009): Sonderauswertung der CVTS3-Zusatzerhebung 2008, Bonn

Bundesinstitut für Berufsbildung (BIBB)/Bundesanstalt für Arbeitsschutz und Arbeitsmedizin (BAuA) (2006): BIBB/BAuA-Erwerbstätigenbefragung 2006 - Arbeit und Beruf im Wandel, Erwerb und Verwertung beruflicher Qualifikationen, Bonn Bundesministerium für Bildung und Forschung (BMBF) (2011): Weiterbildungsverhalten in Deutschland. AES 2010 Trendbericht, Bonn/Berlin Bundesministerium für Familie, Senioren, Frauen und Jugend (BMFSFJ)

(2005): Fünfter Bericht zur Lage der älteren Generation in der Bundesrepublik Deutschland. Potenziale des Alters in Wirtschaft und Gesellschaft. Der Beitrag älterer Menschen zum Zusammenhalt der Generationen, Bericht der Sachverständigenkommission an das BMFSFJ, Berlin

Commerzbank AG (Hrsg.) (2009): Abschied vom Jugendwahn? Unternehmerische Strategien für den demographischen Wandel, Frankfurt a. M.

Dabrowski, H./Neumann, U./Schauer, H./Sperling, H.-J. (1977): Der Lohnrahmentarifvertrag II in der betrieblichen Praxis, Forschungsberichte des SOFI, Göttingen

Deutsche Angestellten Krankenkasse (DAK) (Hrsg.) (2011): Gesundheitsreport 2011. Analyse der Arbeitsunfähigkeitsdaten. Schwerpunktthema: Wie gesund sind junge Arbeitnehmer?, Hamburg

Deutsche Rentenversicherung (DRV) Bund (2011): Rentenzugänge 2010; Renten nach SGB IV insgesamt, Tabelle 012.00 Z Verteilung nach Rentenarten sowie nach Berufen (Tätigkeitsschlüssel) des Versicherten vor Rentenbeginn, Datenfernabfrage, Berlin 
DGB-Index Gute Arbeit GmbH (2009): Datenbestand der Repräsentativerhebung 2009, Berlin

Frehmel, T. (2011): Zwischen Gesetz und Tarif. Altersübergänge als Gestaltungsaufgabe, in: Deutsches Zentrum für Altersfragen (Hrsg.): Informationsdienst Altersfragen 38 (4), S. 9-13

Freidank, J./Grabbe, J./Kädtler, J./Tullius, K. (2011): Altersdifferenzierte und alternsgerechte Betriebs- und Tarifpolitik. Eine Bestandsaufnahme betriebspolitischer und tarifvertraglicher Maßnahmen zur Sicherung der Beschäftigungsfähigkeit, hrsg. von der Bundesanstalt für Arbeitsschutz und Arbeitsmedizin (BAuA) und der Geschäftsstelle der Initiative Neue Qualität der Arbeit (INOA), INQA-Bericht (42), Dortmund/Berlin

Fuchs, J./Zika, G. (2010): Demografie gibt die Richtung vor. Arbeitsmarktbilanz bis 2025, in: IAB-Kurzbericht (12), Juni, Nürnberg

Hans-Böckler-Stiftung (HBS) (2009): Demographie im Wandel. Impulse für eine alternsgerechte Erwerbsarbeit, Düsseldorf

Heien, T./Kowalski, I./Leve, V./Naegele, G./Rockhoff, M./Sporket, M. (2008):

"Rente mit 67" - Voraussetzungen für die Weiterarbeitsfähigkeit älterer Arbeitnehmerinnen. Projektbericht für die Initiative Neue Qualität der Arbeit (INQA), Dortmund

Holler, M./Trischler, F. (2010): Gute Erwerbsbiographien. Arbeitspapier 3: Einflussfaktoren auf die Arbeitsfähigkeit. Der Einfluss belastender Arbeitsbedingungen auf die Gesundheit und die Arbeitsfähigkeit bis zum Rentenalter, Stadtbergen

Ilmarinen, J./Tempel, J. (2002): Arbeitsfähigkeit 2010. Was können wir tun, damit Sie gesund bleiben?, Hamburg

Initiative Neue Qualität der Arbeit (INOA) (2005): Demographischer Wandel und Beschäftigung. Plädoyer für neue Unternehmensstrategien (Memorandum), Dortmund

Kädtler, J. (2010): Finanzmarktkapitalismus und Finanzmarktrationalität., in: Böhle, F./Voß, G. G. Wachtler, G. (Hrsg.): Handbuch Arbeitssoziologie, Wiesbaden, S. 619-639

Katenkamp, O./Martens, H./Georg, A. (2011): Evaluation des Tarifvertrags „Demografischer Wandel" in der Eisen- und Stahlindustrie. Abschlussbericht für die Hans-Böckler-Stiftung, Dortmund

Kaufmann, F.-X. (2005): Schrumpfende Gesellschaft. Vom Bevölkerungsrückgang und seinen Folgen, Bonn

Kistler, E. (2008): „Alternsgerechte Erwerbsarbeit“. Ein Überblick über den Stand von Wissenschaft und Praxis, hrsg. von der Hans Böckler Stiftung, Böckler Forschungsmonitoring (7), Düsseldorf

Kocka, J./Staudinger, U. (Hrsg.) (2009): Gewonnene Jahre. Empfehlungen der Akademiengruppe Altern in Deutschland, Stuttgart

Kuhlmann, M. (2009): Perspektiven der Arbeitspolitik nach der Krise: Entwicklungslinien und Handlungsbedingungen, in: WSI-Mitteilungen 62 (12),

S. 675-682, http://www.boeckler.de/wsi_2885.htm

Latniak, E./Voss-Dahm, D./Elsholz, U./Gottwald, M./Gerisch, S. (2010): Umsetzung demografiefester Personalpolitik in der Chemischen Industrie. Inhaltliche und prozessuale Analyse betrieblicher Vorgehensweisen, Abschlussbericht für die Hans-Böckler-Stiftung, Düsseldorf

Leber, U./Möller, I. (2008): Weiterbildungsbeteiligung ausgewählter Personengruppen, in: Schmollers Jahrbuch 128, S. 405-429

Lorenz, F./Schneider, G. (Hrsg.) (2008): Alternsgerechtes Arbeiten. Der demografische Wandel in den Belegschaften, Hamburg

Morschhäuser, M. (2006): Reife Leistung. Personal- und Qualifizierungspolitik für die künftige Altersstruktur, Forschung aus der Hans-Böckler Stiftung, Bd. 72, Berlin

Matthäi, I./Morschhäuser, M. (2009): Länger arbeiten in gesunden Organisationen. Praxishilfe zur alternsgerechten Arbeitsgestaltung in Industrie, Handel und Öffentlichem Dienst, Saarbrücken

Moraal, D./Lorig, B./Schreiber, D./Azeez, U. (2009): Ein Blick hinter die Kulissen der betrieblichen Weiterbildung in Deutschland. Daten und Fakten der nationalen CVTS3-Zusatzerhebung, BIBB-Report (7), Bonn

Öztürk, H. (2009): Weiterbildungsbeteiligung von Menschen mit Migrationshintergrund in Deutschland, in: Aus Politik und Zeitgeschichte (5), S. 24-30 Reindl, J. (2009): Die Abschaffung des Alters. Eine Kritik des optimistischen Alternsparadigmas, in: Leviathan 37 (1), S. 160-172

Schack, A./Volkwein, C. (Hrsg.) (2010): Der Demografie-Tarifvertrag in der Hessischen Praxis, Wiesbaden
Schumann, M. (1998): Frisst die Shareholder-Value-Ökonomie die Modernisierung der Arbeit?, in: Hirsch-Kreinsen, H./Wolf, H. (Hrsg.): Arbeit, Gesellschaft, Kritik, Berlin, S. 19-30

Statistisches Bundesamt (2009): Erwerbsbeteiligung älterer Arbeitnehmer, Wiesbaden

Statistisches Bundesamt/Wissenschaftszentrum Berlin für Sozialforschung (WZB) (2011): Datenreport 2011. Ein Sozialbericht für die Bundesrepublik Deutschland, Bd. I., Bundeszentrale für politische Bildung, Bonn

Trischler, F./Kistler, E. (2010): Gute Erwerbsbiographien. Arbeitspapier 2: Arbeitsbedingungen und Erwerbsverlauf, Internationales Institut für Empirische Sozialökonomie, Hans-Böckler-Stiftung, Stadtbergen/Düsseldorf

Windolf, P. (2005): Was ist Finanzmarkt-Kapitalismus?, in: Windolf, P. (Hrsg.): Finanzmarkt-Kapitalismus. Analysen zum Wandel von Produktionsregimen, Wiesbaden, S. 20-57

Wolf, E. (Hrsg.) (1985): Schichtarbeit : Umsetzung arbeitswissenschaftlicher Erkenntnisse für Schichtarbeiter und Interessenvertreter: Projekt PROSA. Frankfurt a. M./New York

\section{AUTOREN}

KNUT TULLIUS, Dr., ist wissenschaftlicher Mitarbeiter am Soziologischen Forschungsinstitut (SOFI) an der Georg-August-Universität Göttingen. Arbeitsschwerpunkte: Arbeits- und Industriesoziologie, Weiterbildung, Tarifpolitik.

knut.tullius@sofi.uni-goettingen.de

JOHANNES FREIDANK, Dipl. Pol., war bis 31.12.2010 wissenschaftlicher Mitarbeiter an der Universität Kassel, Fachgebiet Politisches System der Bundesrepublik Deutschland.

jfreidank@gmx.de

JOHANNES GRABBE, M.A., war bis 31.12.2010 wissenschaftlicher Mitarbeiter an der Universität Kassel, Fachgebiet Politisches System der Bundesrepublik Deutschland, seit Juni 2011 ist er beim Vorstand des DGB-Bezirks Niedersachsen, Bremen, Sachsen-Anhalt tätig.

johannes.grabbe@dgb.de

JÜRGEN KÄDTLER, Prof. Dr., ist Direktor des SOFI und außerplanmäßiger Professor an der Georg-August-Universität Göttingen. Arbeitsschwerpunkte: Industrielle Beziehungen, Tarifpolitik, Globalisierung, Soziologie der Finanzmärkte.

juergen.kaedtler@sofi.uni-goettingen.de

WOLFGaNG SCHRoEdeR, Prof. Dr., Universität Kassel. Arbeitsschwerpunkte: Wandel von Politik und Ökonomie in Deutschland und Europa, Vergleichende Wohlfahrtsstaatsforschung, Parteien- und Organisationsforschung.

wolfgang.schroeder@uni-kassel.de 